\title{
Epidemic Spreading with Heterogeneous Awareness on Human Networks
}

\author{
Yanling Lu, ${ }^{1}$ Guoping Jiang, ${ }^{2}$ and Zhengxin Wang ${ }^{1}$ \\ ${ }^{1}$ College of Science, Nanjing University of Posts and Telecommunications, Nanjing 210023, China \\ ${ }^{2}$ College of Automation, Nanjing University of Posts and Telecommunications, Nanjing 210023, China
}

Correspondence should be addressed to Zhengxin Wang; zhengxinwang2008@gmail.com

Received 5 December 2016; Accepted 2 February 2017; Published 4 April 2017

Academic Editor: Haipeng Peng

Copyright (c) 2017 Yanling Lu et al. This is an open access article distributed under the Creative Commons Attribution License, which permits unrestricted use, distribution, and reproduction in any medium, provided the original work is properly cited.

The spontaneous awareness behavioral responses of individuals have a significant impact on epidemic spreading. In this paper, a modified Susceptible-Alert-Infected-Susceptible (SAIS) epidemic model with heterogeneous awareness is presented to study epidemic spreading in human networks and the impact of heterogeneous awareness on epidemic dynamics. In this model, when susceptible individuals receive awareness information about the presence of epidemic from their infected neighbor nodes, they will become alert individuals with heterogeneous awareness rate. Theoretical analysis and numerical simulations show that heterogeneous awareness can enhance the epidemic threshold with certain conditions and reduce the scale of virus outbreaks compared with no awareness. What is more, for the same awareness parameter, it also shows that heterogeneous awareness can slow effectively the spreading size and does not delay the arrival time of epidemic spreading peak compared with homogeneous awareness.

\section{Introduction}

Epidemic spreading has a rich research history and attracted a lot of attention and interest in academia field. Many epidemic models have been studied, including Susceptible-Infected (SI) model [1], Susceptible-Infected-Susceptible (SIS) model [2], and Susceptible-Infected-Removed (SIR) model [3]. The dynamical behavior of epidemics on homogenous networks [4] and heterogeneous networks [5] has been studied by many researchers. Pastor-Satorras and Vespignani studied SIS epidemic model with power-law degree distributions on scale-free (SF) networks, showing that the epidemic threshold vanishes on an infinitely large scale-free networks [6] and remains to be low and decreases with increasing network size in the finite size scale-free networks [7]. In human networks, individuals are generally not evenly distributed but are of heterogeneous distribution. In this paper, we mainly concentrate on the spreading behavior in scale-free networks.

With the rapid development of network technology, the more the channels of obtaining information for people are, the faster the speed of accessing information will be. When people obtain more news about the epidemic, they will appropriately change their behavior in order to prevent infection, such as vaccination [8] and seeking medical services [9]. In the process of epidemic spreading, many susceptible individuals are aware of the potential risks of becoming infected and change their behavior to reduce the possibility of infection $[10,11]$, and the awareness behavior can spread though contact or public media and other tools in the crowd $[12,13]$. In turn, changing of human behavior in the process of epidemic spreading also influences epidemic spreading [1420]. For example, Funk et al. [13] introduced awareness on homogeneous networks to investigate the spread of awareness and its impact on epidemic outbreaks. Wu et al. [16] focused on the three forms of awareness and explored the impact of them on epidemic spreading in a finite scale-free network. Lu et al. [17] and Gong et al. [18] studied epidemic spreading on scale networks considering awareness and individual vigilance, respectively. In these papers, awareness interacts with epidemic and the existence of awareness can significantly enhance the epidemic threshold or reduce the scale of virus outbreaks.

The above literature considers the effect of individual awareness behavioral responses on the infection rate. The 
change of individual awareness behavioral also affects the individual state. For example, individuals go to the removed state when they receive vaccination [21]. Recently, Sahneh and Scoglio [22] proposed a Susceptible-Alert-InfectedSusceptible (SAIS) model in human networks by introducing an "alert" class. Their results suggest that awareness can be considered as a strategy of controlling epidemic spreading. Built upon the SAIS model, Sahneh et al. [23, 24] further studied a threshold for preventive behavioral responses to suppress epidemic spreading and investigated optimal information dissemination to boost the resilience of the agent population against the spreading. The interplay between epidemic spreading and human behavior causes the richer network dynamics in complex networks [25-28]. Therefore, introducing individual behavioral responses to the dynamic of epidemic spreading is very important.

In this paper, susceptible nodes are aware of the risks of becoming infected and can receive awareness about the presence of epidemic from their infected neighbor nodes. In general, when a susceptible node receives awareness, it will become an alert node with certain probability. But the number of infected neighbor nodes of each susceptible node is different in the actual world. Therefore, the alert rate from a susceptible node to an alert node should be different. This paper considers the heterogeneous awareness behavioral responses of individuals, so the probability from susceptible nodes to alert nodes is varied. The role of heterogeneity behavioral responses in complex networks is relatively less studied. In this study, we will investigate the variety of the epidemic threshold between the modified model and standard model and analyze the effect of heterogeneous awareness for epidemic spreading.

The rest of this paper is organized as follows. In Section 2, we propose the modified SAIS model with heterogeneous awareness behavioral responses on scale-free networks. In Section 3, we will analyze the modified SAIS model and deduce the mathematical expression of the epidemic threshold. Some numerical simulations are done in Section 4 . Finally, Section 5 concludes the paper.

\section{The SAIS Model with Heterogeneous Awareness}

The SAIS epidemic model introduces human behavioral responses and adds a new compartment to the class SIS model. Each individual is represented by a node of the network and the edges are the physical interactions between individuals along which the infection spreads on scale-free networks. When susceptible nodes contact with their infected neighbor nodes, they may become alert nodes with a certain probability. Let $S_{k}(t), A_{k}(t)$, and $I_{k}(t)$ be the densities of susceptible, alert, and infected nodes of degree $k$ at time $t$. These variables satisfy the following normalization condition:

$$
S_{k}(t)+A_{k}(t)+I_{k}(t)=1 \text {. }
$$

The SAIS epidemic model has been clearly described in Section 4. At each time step, each susceptible node is infected with infection probability $\beta_{0}$ if it is connected to an infected

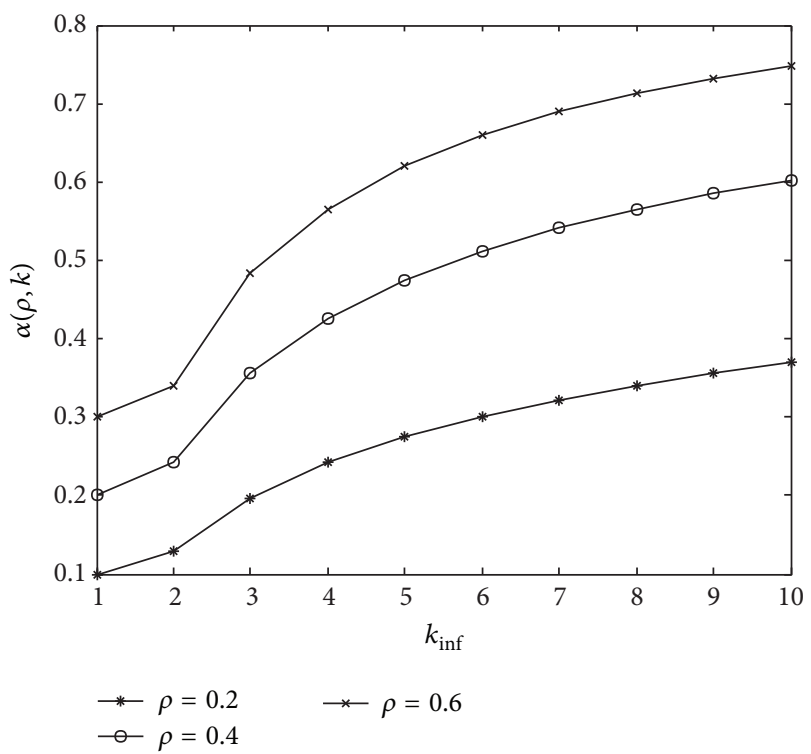

Figure 1: Awareness rate function $\alpha(\rho, k)$ with different $\rho \in(0,1)$.

individual; on the other hand, each infected node recovers and becomes susceptible node with the recovery rate $\delta$ in the SAIS epidemic model. Specifically, susceptible nodes can receive awareness information from infected neighbor nodes and may become alert nodes with the alerting rate $\alpha$ when susceptible nodes contact with its infected neighbor nodes. Both susceptible and alert nodes can potentially be infected in the SAIS model. But, the infection rate for the alert nodes compared with the susceptible nodes is low. That is to say, an alert node is infected with infection probability $0<\beta_{\alpha}<\beta_{0}$. Therefore $\lambda=\beta_{0} / \delta$ (without lack of generality, we set $\delta=1$ ) is considered as an effective spreading rate. In order to simplify calculation, the translation rate from the susceptible node to the alert node is usually set as constant. But in fact, the awareness information from the neighbor nodes is different for each susceptible node. So, we consider the heterogeneous awareness behavioral responses of individual.

Generally, the higher the degree a node has, the stronger the awareness will be. So, the awareness function can be supposed as follows:

$$
\alpha(\rho, k)= \begin{cases}1-k_{\mathrm{inf}}^{-\rho}, & k_{\mathrm{inf}}>1, \\ \frac{\rho}{2}, & k_{\mathrm{inf}}=1,\end{cases}
$$

where the awareness constant $\rho \in(0,1)$. Let $k_{\text {inf }}$ be the total number of infected neighbor nodes of susceptible nodes of degree $k$. The awareness function of susceptible nodes is an increasing function of $k_{\text {inf }}$ and awareness constant $\rho$. An example of awareness rate function $\alpha(\rho, k)$ is shown in Figure 1. 
In summary, the differential equations for modified SAIS model based on the mean field theory are given, as follows:

$$
\begin{aligned}
& \frac{d S_{k}(t)}{d t}=-\beta_{0} k S_{k}(t) \Theta(t)-\alpha(\rho, k) k S_{k}(t) \Theta(t) \\
&+\delta I_{k}(t), \\
& \frac{d I_{k}(t)}{d t}= \beta_{0} k S_{k}(t) \Theta(t)+\beta_{\alpha} k A_{k}(t) \Theta(t)-\delta I_{k}(t), \\
& \frac{d A_{k}(t)}{d t}=\alpha(\rho, k) k S_{k}(t) \Theta(t)-\beta_{\alpha} k A_{k}(t) \Theta(t),
\end{aligned}
$$

where $0 \leq \Theta(t) \leq 1$ denotes the average probability of any given link points to an infected node. Considering nonhomogeneity of uncorrelated scale-free networks, $\Theta(t)$ satisfies the relation

$$
\Theta(t)=\frac{1}{\bar{k}} \sum_{k} k P(k) I_{k}(t),
$$

where $\bar{k}=\sum_{k} k P(k)$.

When $\alpha(\rho, k)=0$ and $\beta_{\alpha}=0$, the model becomes classical SIS model on scale-free networks. The following differential equation for SIS epidemic model is obtained:

$$
\frac{d I_{k}^{\prime}(t)}{d t}=\beta_{0} k\left(1-I_{k}^{\prime}(t)\right) \Theta^{\prime}(t)-\delta I_{k}^{\prime}(t),
$$

where $I_{k}^{\prime}(t)$ are the densities of infected nodes of degree $k$ at time $t$ and $\Theta^{\prime}(t)=(1 / \bar{k}) \sum_{k} k P(k) I_{k}^{\prime}(t)$.

\section{Theoretical Analysis of the SAIS Epidemic Model}

With the normalization condition (1), the differential equations (3) can be simplified as

$$
\begin{aligned}
\frac{d I_{k}(t)}{d t}= & \beta_{0} k\left(1-I_{k}(t)-A_{k}(t)\right) \Theta(t) \\
& +\beta_{\alpha} k A_{k}(t) \Theta(t)-\delta I_{k}(t), \\
\frac{d A_{k}(t)}{d t}= & \alpha(\rho, k) k\left(1-I_{k}(t)-A_{k}(t)\right) \Theta(t) \\
& -\beta_{\alpha} k A_{k}(t) \Theta(t) .
\end{aligned}
$$

3.1. Infected Intensity. Beginning with the same initial infected densities of nodes $I_{k}\left(t_{0}\right)=I_{k}^{\prime}\left(t_{0}\right), k \in\{1, \ldots, N\}$, for the SIS model (5) and SAIS model (6) and (7).

Equation (6) can be rewritten as [22]

$$
\begin{aligned}
\frac{d I_{k}(t)}{d t}= & \beta_{0} k\left(1-I_{k}(t)\right) \Theta(t) \\
& -\left(\beta_{0}-\beta_{\alpha}\right) k A_{k}(t) \Theta(t)-\delta I_{k}(t) .
\end{aligned}
$$

Since $0<\beta_{\alpha}<\beta_{0}$, therefore $\left(\beta_{0}-\beta_{\alpha}\right) k A_{k}(t) \Theta(t)$ is a nonnegative term. Furthermore, beginning with the same initial infected densities of nodes $I_{k}\left(t_{0}\right)=I_{k}^{\prime}\left(t_{0}\right)$, we can get inequality [22], as follows:

$$
\frac{d I_{k}\left(t_{0}\right)}{d t} \leq \frac{d I_{k}^{\prime}\left(t_{0}\right)}{d t} .
$$

According to (9) and definition of the derivative, there exists $t_{T}$ so that [22]

$$
I_{k}(t) \leq I_{k}^{\prime}(t), \quad k \in\{1, \ldots, N\} \quad \forall t \in\left[t_{0}, t_{T}\right] .
$$

Inequality (10) can extend to

$$
I_{k}(t) \leq I_{k}^{\prime}(t), \quad k \in\{1, \ldots, N\} \quad \forall t \in\left[t_{0}, \infty\right) .
$$

Next, we will prove that inequality (11) holds using reduction to absurdity [22]. Assume that there exists $t_{1}>t_{0}$, so that (10) holds for $t_{T}=t_{1}$ but it is not true for every $t_{T}>t_{1}$. Obviously, when $t=t_{1}$,

$$
\exists k \in\{1, \ldots, N\} \text { so that }
$$

$$
I_{k}\left(t_{1}\right)=I_{k}^{\prime}\left(t_{1}\right), \frac{d I_{k}\left(t_{1}\right)}{d t}>\frac{d I_{k}^{\prime}\left(t_{1}\right)}{d t} .
$$

According to (8), (10), and (12), we have

$$
\begin{aligned}
\frac{d I_{k}\left(t_{1}\right)}{d t}= & \beta_{0} k\left(1-I_{k}\left(t_{1}\right)\right) \Theta\left(t_{1}\right) \\
& -\left(\beta_{0}-\beta_{\alpha}\right) k A_{k}\left(t_{1}\right) \Theta\left(t_{1}\right)-\delta I_{k}\left(t_{1}\right) \\
\leq & \beta_{0} k\left(1-I_{k}\left(t_{1}\right)\right) \Theta\left(t_{1}\right)-\delta I_{k}\left(t_{1}\right) \\
= & \beta_{0} k\left(1-I_{k}^{\prime}\left(t_{1}\right)\right) \Theta\left(t_{1}\right)-\delta I_{k}^{\prime}\left(t_{1}\right) \\
\leq & \beta_{0} k\left(1-I_{k}^{\prime}\left(t_{1}\right)\right) \Theta^{\prime}\left(t_{1}\right)-\delta I_{k}^{\prime}\left(t_{1}\right) \\
= & \frac{d I_{k}^{\prime}\left(t_{1}\right)}{d t}
\end{aligned}
$$

Therefore, (13) contradicts with (12). In other words, inequality (10) holds for any $t_{T} \in\left[t_{0}, \infty\right)$. In short, inequality (11) holds.

According to the expression of infected intensity $I(t)=$ $\sum_{k} P(k) I_{k}(t)$, we have the following conclusion.

Theory 1. Beginning with the same initial infected densities of nodes $I_{k}\left(t_{0}\right)=I_{k}^{\prime}\left(t_{0}\right), k \in\{1, \ldots, N\}$, the infected intensity of SIS epidemic model (5) always dominates these of the SAIS epidemic model (6) and (7); that is,

$$
I(t) \leq I^{\prime}(t), \quad \forall t \in\left[t_{0}, \infty\right) .
$$

3.2. Epidemic Threshold. Define $I_{k}$ and $A_{k}$ as infected densities and alert densities of nodes in the steady stay, respectively. We have

$$
\begin{array}{r}
\beta_{0} k\left(1-I_{k}-A_{k}\right) \Theta(p)+\beta_{\alpha} k A_{k} \Theta(p)-\delta I_{k}=0, \\
\alpha(\rho, k) k\left(1-I_{k}-A_{k}\right) \Theta(p)-\beta_{\alpha} k A_{k}(t) \Theta(p)=0 .
\end{array}
$$


We can derive from (15)

$$
\begin{aligned}
I_{k} & =\frac{\beta_{0} k \Theta(p)-\left(\beta_{0}-\beta_{\alpha}\right) k A_{k} \Theta(p)}{\beta_{0} k \Theta(p)+\delta}, \\
A_{k} & =\frac{\alpha(\rho, k)\left(1-I_{k}\right)}{\beta_{\alpha}+\alpha(\rho, k)} .
\end{aligned}
$$

By substituting (16) in (4), we can get

$$
\begin{aligned}
\Theta & (p) \\
& =\frac{1}{\bar{k}} \sum_{k} \frac{\left(\beta_{0}+\alpha(\rho, k)\right) \beta_{\alpha} k^{2} p(k) \Theta(p)}{\left(\beta_{0}+\alpha(\rho, k)\right) \beta_{\alpha} k \Theta(p)+\delta\left(\beta_{\alpha}+\alpha(\rho, k)\right)} \\
& =F(\Theta(p)) .
\end{aligned}
$$

Obviously $\Theta(p)=0$ is a trivial solution of (17). To an epidemic outbreak, there must be a nonzero solution of $\Theta(p)$, satisfying

$$
\left.\frac{d F(\Theta(p))}{d \Theta(p)}\right|_{\Theta(p)=0} \geq 1
$$

Equation (18) can be rewritten as

$$
\frac{\beta_{0}}{\delta} \frac{\beta_{\alpha}}{\bar{k}} \overline{\frac{k^{2}}{\beta_{\alpha}+\alpha(\rho, k)}}+\frac{\beta_{\alpha}}{\delta} \frac{1}{\bar{k}} \frac{\overline{\alpha(\rho, k) k^{2}}}{\beta_{\alpha}+\alpha(\rho, k)} \geq 1 .
$$

Let $\lambda_{c}$ be the epidemic threshold of scale-free networks. We can obtain the mathematical expression of the epidemic threshold by (20), as follows:

$$
\begin{aligned}
\lambda_{c} & =\frac{\beta_{0}}{\delta} \\
& =\frac{\left(\bar{k} / \beta_{\alpha}\right)-(1 / \delta) \overline{\left(\alpha(\rho, k) k^{2}\right) /\left(\beta_{\alpha}+\alpha(\rho, k)\right)}}{\overline{k^{2} /\left(\beta_{\alpha}+\alpha(\rho, k)\right)}} .
\end{aligned}
$$

If $\alpha(\rho, k)=\rho$ is a constant, that is to say, the alerting rate from susceptible nodes to alert nodes is a constant, we can get the epidemic threshold $\lambda_{c_{2}}$ of SAIS model with homogeneous awareness:

$$
\lambda_{c_{2}}=\frac{\bar{k}}{\overline{k^{2}}}+\frac{\rho}{\beta_{\alpha}}\left(\frac{\bar{k}}{\overline{k^{2}}}-\frac{\beta_{\alpha}}{\delta}\right) .
$$

As everyone knows, the epidemic threshold of SIS epidemic model is $\lambda_{c_{1}}=\bar{k} / \overline{k^{2}}$ on scale-free networks. If $\alpha(\rho, k)=$ $\rho$ and $\bar{k} / \overline{k^{2}}>\beta_{\alpha} / \delta$, then $\lambda_{c_{2}}>\lambda_{c_{1}}$.

If $\alpha(\rho, k)$ adopts piecewise awareness function (2), then $\rho / 2 \leq \alpha(\rho, k)<1$. Furthermore, we can obtain that $\left(k^{2} /\left(\beta_{\alpha}+\right.\right.$ $1))<\left(k^{2} /\left(\beta_{\alpha}+\alpha(\rho, k)\right)\right)<\left(2 k^{2} /\left(2 \beta_{\alpha}+\rho\right)\right)$ and $\left(\rho k^{2} /\left(2 \beta_{\alpha}+\right.\right.$ $\rho))<\left(\alpha(\rho, k) k^{2} /\left(\beta_{\alpha}+\alpha(\rho, k)\right)\right)<\left(k^{2} /\left(\beta_{\alpha}+1\right)\right)$.

Thus, we can get the epidemic threshold $\lambda_{c_{3}}$ of SAIS model with heterogeneous awareness:

$$
m<\lambda_{c_{3}}<n
$$

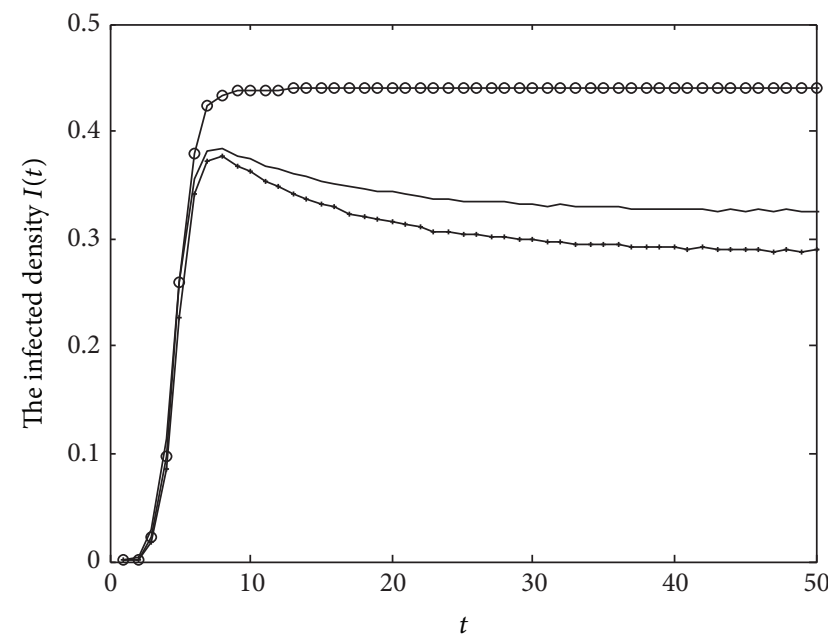

FIGURE 2: The infected density $I(t)$ for SIS model and SAIS model with different heterogeneous awareness $\rho$.

where $m=\left(\bar{k} / \overline{k^{2}}\right)+\left((\rho / 2) / \beta_{\alpha}\right)\left(\bar{k} / \overline{k^{2}}-\beta_{\alpha}\left(2 \beta_{\alpha}+\rho\right) / \delta\left(\beta_{\alpha}+1\right) \rho\right)$ and $n=\bar{k} / \overline{k^{2}}+\left(1 / \beta_{\alpha}\right)\left(\bar{k} / \overline{k^{2}}-\beta_{\alpha}\left(\beta_{\alpha}+1\right) \rho / \delta\left(2 \beta_{\alpha}+\rho\right)\right)$.

It is obvious that $\left(2 \beta_{\alpha}+\rho\right) /\left(\beta_{\alpha}+1\right) \rho>\left(\beta_{\alpha}+1\right) \rho /\left(2 \beta_{\alpha}+\rho\right)$. Therefore, we have the following conclusion.

Theory 2. For the awareness constant $\rho \in(0,1)$, the epidemic threshold of SAIS model with heterogeneous awareness is higher than that of SIS model, that is $\lambda_{c_{3}}>\lambda_{c_{1}}$, under the following condition:

$$
\frac{\bar{k}}{\overline{k^{2}}}>\frac{\beta_{\alpha}\left(2 \beta_{\alpha}+\rho\right)}{\delta\left(\beta_{\alpha}+1\right) \rho} .
$$

Furthermore, we can obtain the following: if the infected rate $\beta_{\alpha}$ and the awareness intensity $\rho$ satisfy the following conditions (24), then $\lambda_{c_{1}}=\langle k\rangle /\left\langle k^{2}\right\rangle>\beta_{\alpha}\left(2 \beta_{\alpha}+\rho\right) / \delta\left(\beta_{\alpha}+1\right) \rho$.

$0<\beta_{\alpha}$

$$
<\frac{-\left(1-\lambda_{c_{1}} \delta\right) \rho+\sqrt{\left[\left(1-\lambda_{c_{1}} \delta\right) \rho\right]^{2}+8 \lambda_{c_{1}} \delta \rho}}{4},
$$

$$
\rho>\frac{2 \beta_{\alpha}^{2}}{\delta\left(\beta_{\alpha}+1\right) \lambda_{c_{1}}-\beta_{\alpha}}, \quad \text { when } \beta_{\alpha}<\frac{\delta \lambda_{c_{1}}}{1-\delta \lambda_{c_{1}}} \text {. }
$$

\section{Simulation Results and Discussion}

In this section, we perform the simulations on an $N$-node BA networks to verify our analysis. The parameters for generating a BA network are as follows: network size $N=1000$, the initial number of nodes $m_{0}=3$, the new number of links for each new node $m=3$, and the average degree $\bar{k}=6$. For SIS epidemic model on this work, the epidemic threshold is $\lambda_{c_{1}} \approx 0.0574$. For each simulation, the infected node is randomly chosen when run begins and the recovery rate is fixed at $\delta=1$.

In Figure 2, the circle line stands for SIS epidemic model, and the solid line and the star line stand for SAIS epidemic 


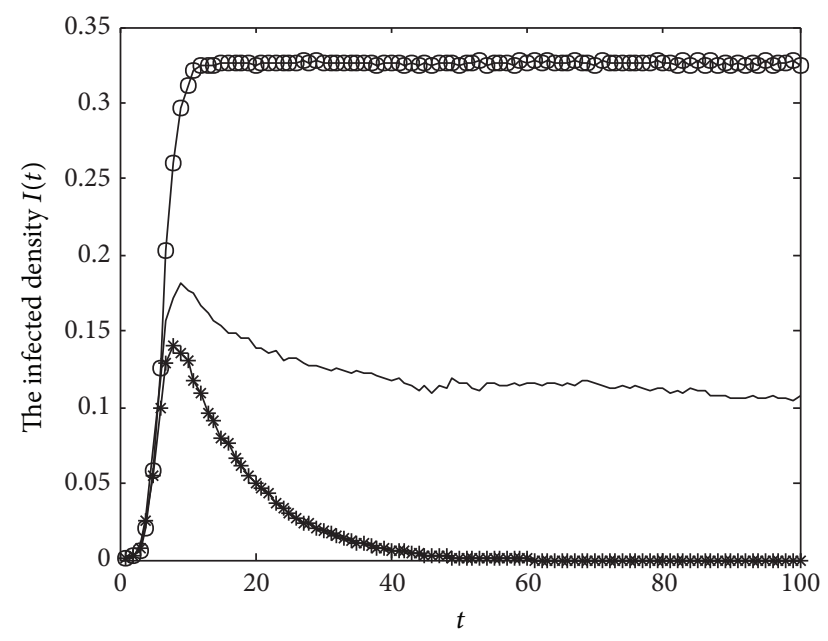

FIGURE 3: The infected density $I(t)$ for SIS model and SAIS model with heterogeneous awareness of different infected rate $\beta_{\alpha}$.

model with different heterogeneous awareness $\rho=0.2$ and $\rho=0.4$, respectively. Set the infected rate $\beta_{0}=0.5$ representing the probability that a susceptible node moves into the infected state and $\beta_{\alpha}=0.04$ representing the probability that an alert node moves into the infected state. Through theoretical analysis, we can obtain that the infected intensity of SIS epidemic model always dominates that of the SAIS epidemic model with heterogeneous awareness, beginning with the same initial infected densities of nodes.

Figure 2 shows that the result of numerical simulation is consistent with the theoretical analysis. That is to say, the introducing of the heterogeneous awareness significant can decrease the infected density, which demonstrates that the SAIS model with heterogeneous awareness is reasonable and consistent with the realistic situation.

In Figure 3, the circle line stands for SIS epidemic model, and the solid lines and the star line stand for SAIS model with heterogeneous awareness in case $\beta_{\alpha}=0.08,0.04$, respectively. Set the infected rate $\beta_{0}=0.3$ representing the probability that a susceptible node moves into the infected state and the awareness parameter $\rho=0.5$ for SAIS model with heterogeneous awareness.

Figure 3 shows that the epidemic of SAIS model is persistent and the epidemic size is reduced in the steady state compared with SIS model when $\beta_{\alpha}=0.08$, and the epidemic dies out asymptotically when $\beta_{\alpha}=0.04$. These imply that the epidemic either dies out or breaks out eventually in relation with $\beta_{\alpha}$ when $\rho>\beta_{0}$.

Figure 4 detailedly shows the effect of $\rho$ for SAIS model with heterogeneous awareness. In Figure 4, the circle, solid, and star line stand for SAIS model with heterogeneous awareness for different awareness parameters $\rho=0.2,0.3,0.4$, respectively. Set the infected rate $\beta_{0}=0.3$ representing the probability that a susceptible node moves into the infected state and $\beta_{\alpha}=0.04$ representing the probability that an alert node moves into the infected state in Figure 4. Analyzing the curves of Figure 4, we can find that the epidemic size decreases with the increasing of awareness parameters $\rho$. We

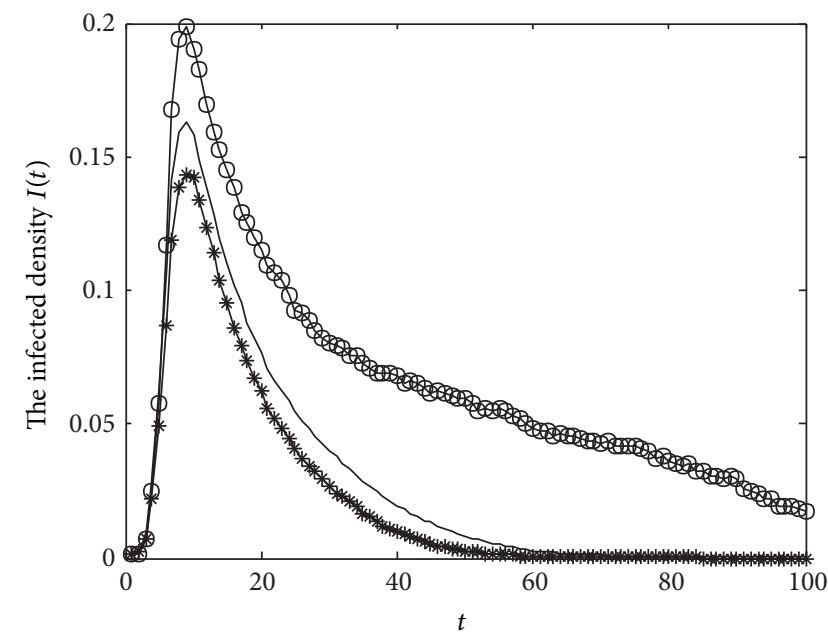

FIGURE 4: The infected density $I(t)$ for SAIS model with different heterogeneous awareness $\rho$.

can also see that the epidemic persists in the steady state when $\beta_{0}>\rho$, and the epidemic dies out asymptotically when $\beta_{0} \leq \rho$ in the condition of $\beta_{\alpha}<\lambda_{c_{1}}$.

In order to more detailedly compare the effect of $\rho$ for SAIS model with awareness, we consider two different cases: homogeneous awareness and heterogeneous awareness. We perform some simulations and get Figure 5. In these figures, the circle line and the star line stand for SAIS model with homogeneous awareness and SAIS model with heterogeneous awareness, respectively. The infected rates $\beta_{0}=0.3$ and $\beta_{\alpha}=0.04$ are both fixed, and the awareness parameters are $\rho=0.2,0.3,0.4$, respectively.

Analyzing the cures in Figures 5(a)-5(c), we can find that heterogeneous awareness can slow effectively the spreading size and does not delay the arrival time of epidemic spreading peak compared with homogeneous awareness, for the same awareness parameter.

\section{Conclusions}

In this paper, we have studied the impact of awareness responses on epidemic spreading in human networks and proposed a modified Susceptible-Alert-Infected-Susceptible model with heterogeneous awareness. In particular, susceptible individuals who are aware of the potential risks of becoming infected can take measures to reduce the possibility of infection. In our new model, susceptible nodes can receive awareness information from infected neighbor nodes and become alert nodes with heterogeneous awareness rate. Theoretical analysis and simulation results have shown that the existence of heterogeneous awareness can significantly reduce the risk of virus outbreak, increase the spreading threshold, and slow down the spreading speed in human networks. In order to better control epidemic spreading on networks, the effects of awareness for epidemic spreading are worth further study. 


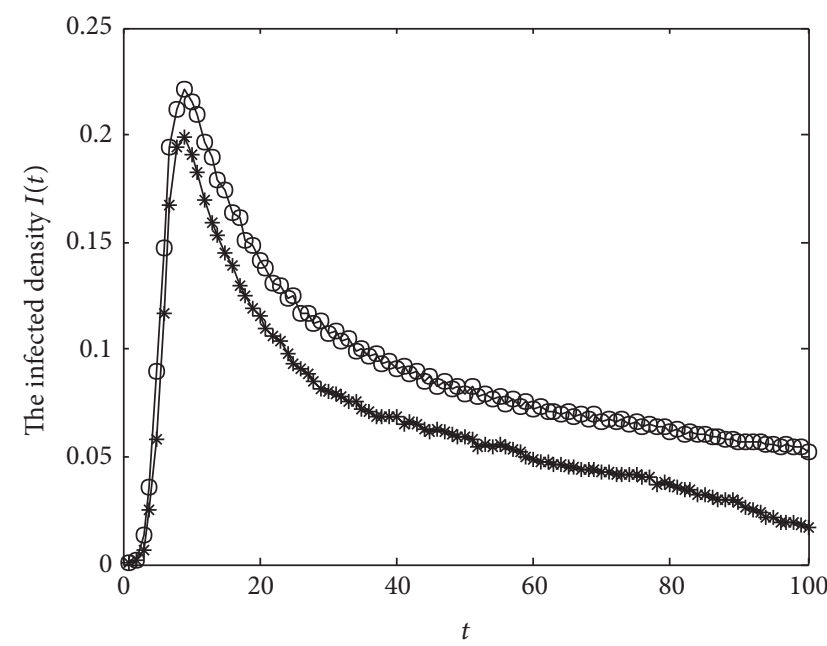

(a) $\rho=0.2$

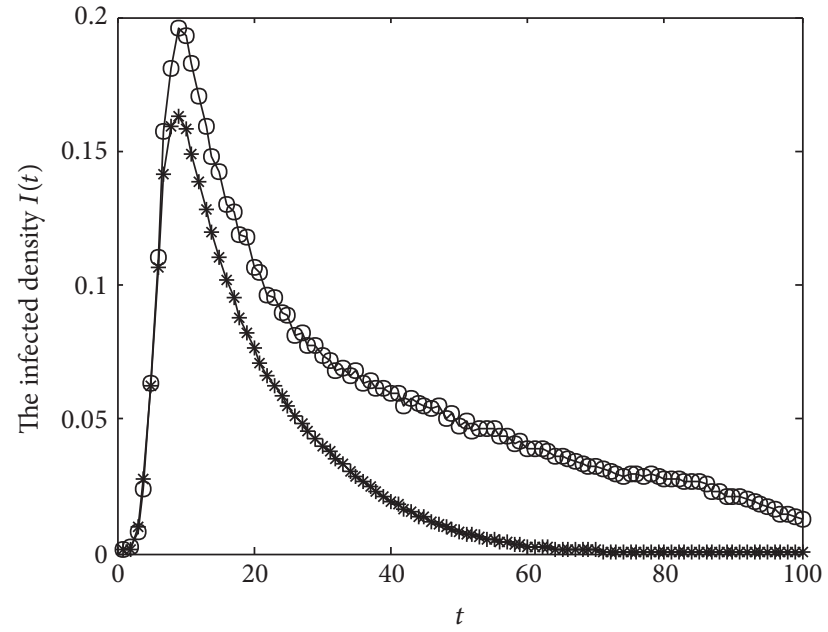

(b) $\rho=0.3$

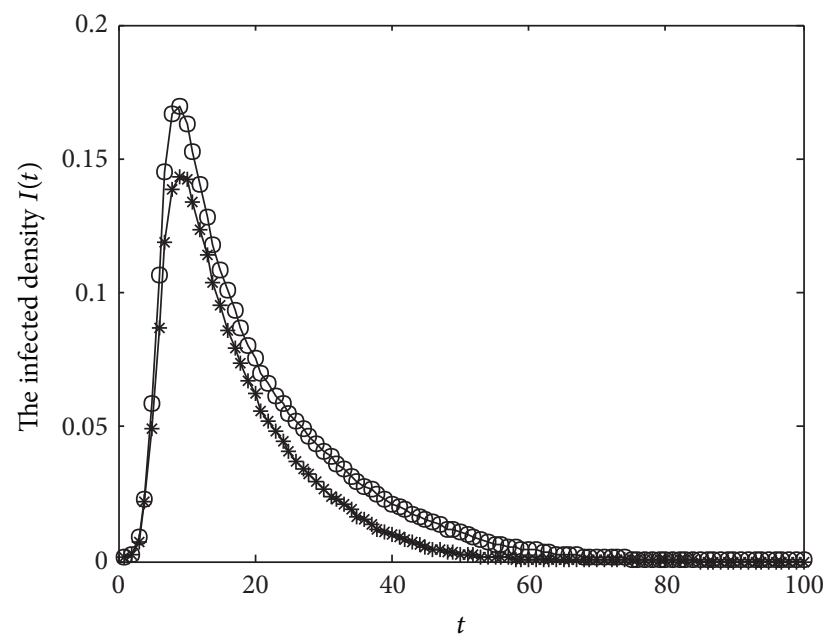

(c) $\rho=0.4$

FIGURE 5: The infected density $I(t)$ for SAIS model with homogeneous and heterogeneous awareness of different awareness $\rho$.

\section{Competing Interests}

The authors declare that there is no conflict of interests regarding the publication of this paper.

\section{Acknowledgments}

This work was supported by the National Natural Science Foundation of China [Grant nos. 61672298 and 61304169]; the Postdoctoral Science Foundation of China [no. 2014M551629]; the Postdoctoral Science Foundation of Jiangsu Province of China [no. 1402086C]; and the Natural and the Nanjing University of Posts and Telecommunications [Grant no. NY215065].

\section{References}

[1] A. Barrat, M. Barthélemy, and A. Vespignani, Dynamical Processes on Complex Networks, Cambridge University Press, Cambridge, UK, 2008.
[2] S. Kwon and Y. Kim, "Epidemic spreading in annealed directed networks: susceptible-infected-susceptible model and contact process," Physical Review E-Statistical, Nonlinear, and Soft Matter Physics, vol. 87, no. 1, Article ID 012813, 2013.

[3] H. Zhang, Z.-H. Guan, T. Li, X.-H. Zhang, and D.-X. Zhang, "A stochastic SIR epidemic on scale-free network with community structure," Physica A. Statistical Mechanics and its Applications, vol. 392, no. 4, pp. 974-981, 2013.

[4] R. Pastor-Satorras and A. Vespignani, "Epidemic dynamics and endemic states in complex networks," Physical Review E, vol. 63, no. 6, Article ID 066117, 2001.

[5] Y. Moreno, R. Pastor-Satorras, and A. Vespignani, "Epidemic outbreaks in complex heterogeneous networks," European Physical Journal B, vol. 26, no. 4, pp. 521-529, 2002.

[6] R. Pastor-Satorras and A. Vespignani, "Epidemic spreading in scale-free networks," Physical Review Letters, vol. 86, no. 14, pp. 3200-3203, 2001.

[7] R. Pastor-Satorras and A. Vespignani, "Epidemic dynamics in finite size scale-free networks," Physical Review E-Statistical, Nonlinear, and Soft Matter Physics, vol. 65, no. 3, Article ID 035108, 2002. 
[8] A. d'Onofrio, P. Manfredi, and E. Salinelli, "Vaccinating behaviour, information, and the dynamics of SIR vaccine preventable diseases," Theoretical Population Biology, vol. 71, no. 3, pp. 301317, 2007.

[9] J. T. F. Lau, X. Yang, H. Y. Tsui, and J. H. Kim, "Impacts of SARS on health-seeking behaviors in general population in Hong Kong," Preventive Medicine, vol. 41, no. 2, pp. 454-462, 2005.

[10] N. Ferguson, "Capturing human behaviour," Nature, vol. 446, no. 7137, Article ID 733, 2007.

[11] M. C. González, C. A. Hidalgo, and A.-L. Barabási, "Understanding individual human mobility patterns," Nature, vol. 453, no. 7196, pp. 779-782, 2008.

[12] I. Z. Kiss, J. Cassell, M. Recker, and P. L. Simon, "The impact of information transmission on epidemic outbreaks," Mathematical Biosciences, vol. 225, no. 1, pp. 1-10, 2010.

[13] S. Funk, E. Gilad, C. Watkins, and V. A. A. Jansen, "The spread of awareness and its impact on epidemic outbreaks," Proceedings of the National Academy of Sciences of the United States of America, vol. 106, no. 16, pp. 6872-6877, 2009.

[14] S. Funk, M. Salathé, and V. A. A. Jansen, "Modelling the influence of human behaviour on the spread of infectious diseases: a review," Journal of the Royal Society Interface, vol. 7, no. 50, pp. 1247-1256, 2010.

[15] S. Funk, E. Gilad, and V. A. Jansen, "Endemic disease, awareness, and local behavioural response," Journal of Theoretical Biology, vol. 264, no. 2, pp. 501-509, 2010.

[16] Q. Wu, X. Fu, M. Small, and X.-J. Xu, "The impact of awareness on epidemic spreading in networks," Chaos: An Interdisciplinary Journal of Nonlinear Science, vol. 22, no. 1, Article ID 013101, 2012.

[17] Y.-L. Lu, G.-P. Jiang, and Y.-R. Song, "Epidemic spreading on a scale-free network with awareness," Chinese Physics B, vol. 21, no. 10, Article ID 100207, 2012.

[18] Y.-W. Gong, Y.-R. Song, and G.-P. Jiang, "Epidemic spreading in scale-free networks including the effect of individual vigilance," Chinese Physics B, vol. 21, no. 1, 2012.

[19] Y.-L. Lu, G.-P. Jiang, and Y.-R. Song, "Stability and bifurcation of epidemic spreading on adaptive network," Acta Physica Sinica, vol. 62, no. 13, Article ID 13020, 2013.

[20] H. Zhang, M. Small, X. Fu, G. Sun, and B. Wang, "Modeling the influence of information on the coevolution of contact networks and the dynamics of infectious diseases," Physica D: Nonlinear Phenomena, vol. 241, no. 18, pp. 1512-1517, 2012.

[21] T. C. Reluga and A. P. Galvani, "A general approach for population games with application to vaccination," Mathematical Biosciences, vol. 230, no. 2, pp. 67-78, 2011.

[22] F. D. Sahneh and C. Scoglio, "Epidemic spread in human networks," in Proceedings of the 50th IEEE Conference on Decision and Control and European Control Conference (CDCECC '11), pp. 3008-3013, Orlando, Fla, USA, December 2011.

[23] F. D. Sahneh, F. N. Chowdhury, and C. M. Scoglio, "On the existence of a threshold for preventive behavioral responses to suppress epidemic spreading," Scientific Reports, vol. 2, article 632, 2012

[24] F. D. Sahneh and C. M. Scoglio, "Optimal information dissemination in epidemic networks," in Proceedings of the 51st IEEE Conference on Decision and Control (CDC '12), pp. 1657-1662, December 2012.

[25] S. Meloni, N. Perra, A. Arenas, S. Gómez, Y. Moreno, and A. Vespignani, "Modeling human mobility responses to the largescale spreading of infectious diseases," Scientific Reports, vol. 1, article 62, 2011.
[26] B. Min, S. Lee, K.-M. Lee, and K.-I. Goh, "Link overlap, viability, and mutual percolation in multiplex networks," Chaos, Solitons and Fractals, vol. 72, pp. 49-58, 2015.

[27] G. Bianconi, "Statistical mechanics of multiplex networks: entropy and overlap," Physical Review E-Statistical, Nonlinear, and Soft Matter Physics, vol. 87, no. 6, Article ID 062806, 2013.

[28] K.-M. Lee, B. Min, and K.-I. Goh, "Towards real-world complexity: an introduction to multiplex networks," The European Physical Journal B, vol. 88, no. 2, article 48, pp. 1-20, 2015. 


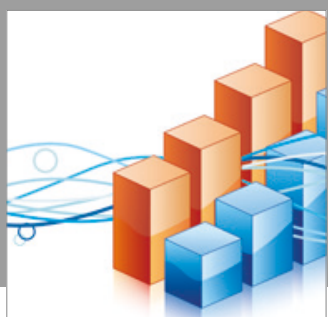

Advances in

Operations Research

vatersals

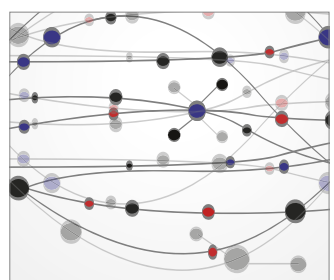

\section{The Scientific} World Journal
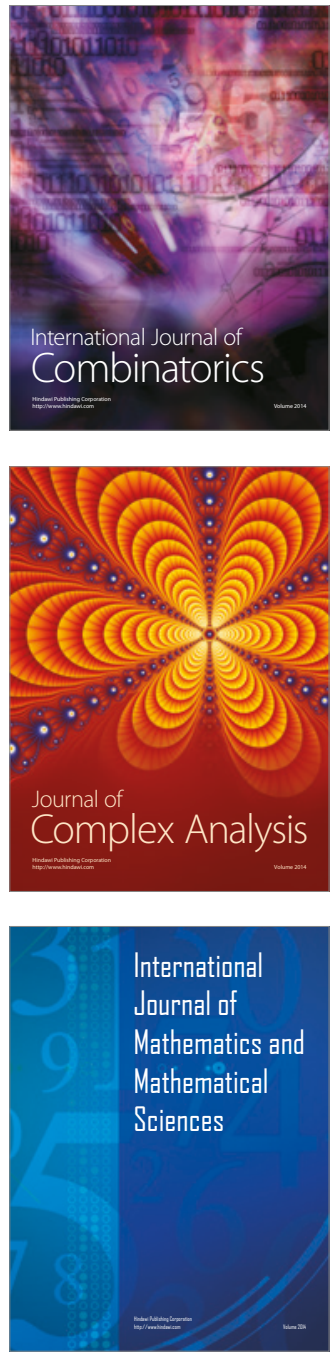
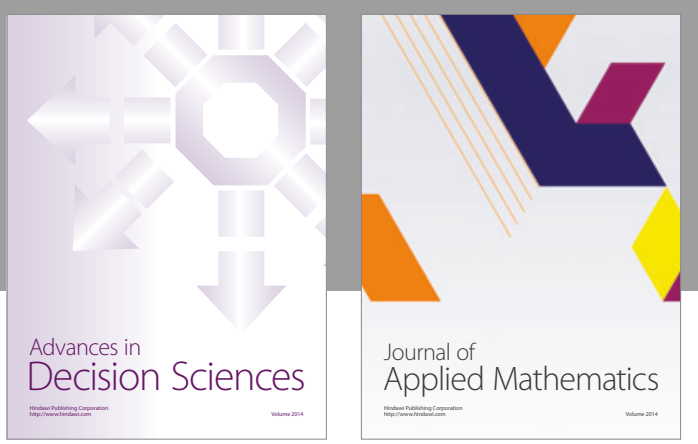

Algebra

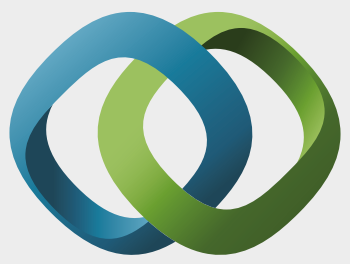

\section{Hindawi}

Submit your manuscripts at

https://www.hindawi.com
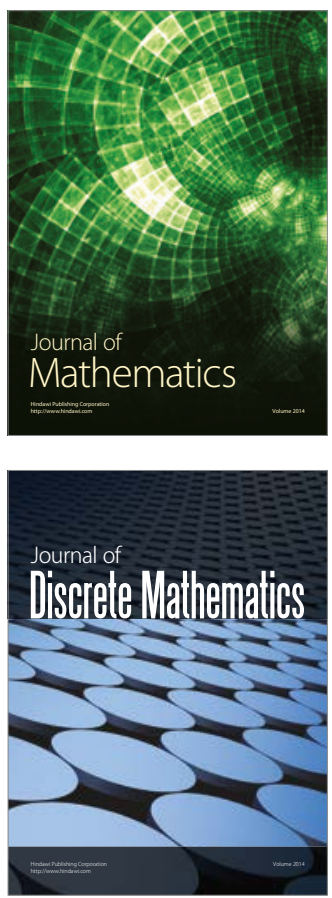

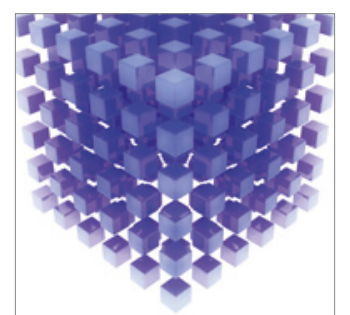

Mathematical Problems in Engineering
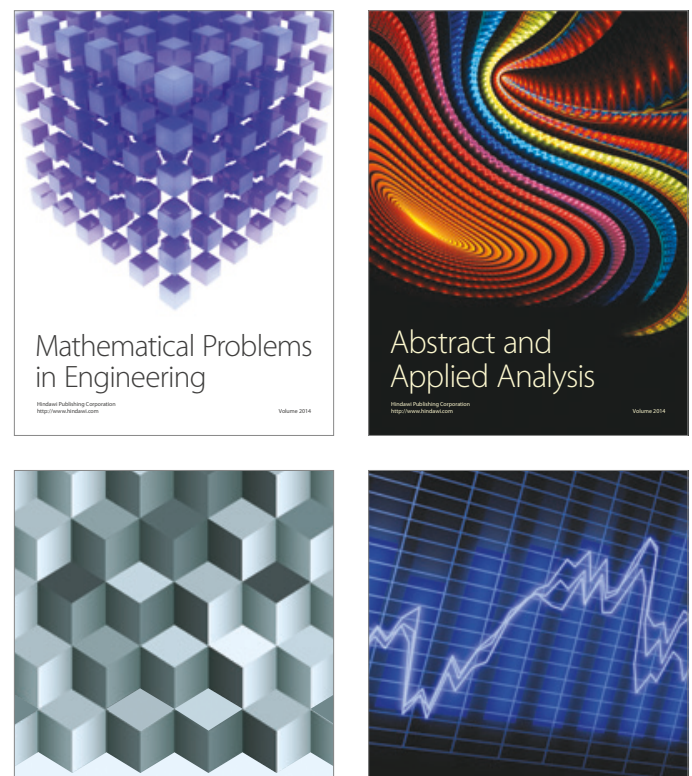

Journal of

Function Spaces

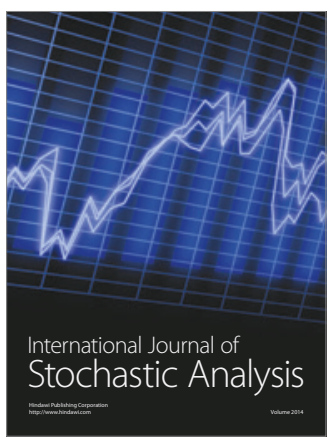

Probability and Statistics
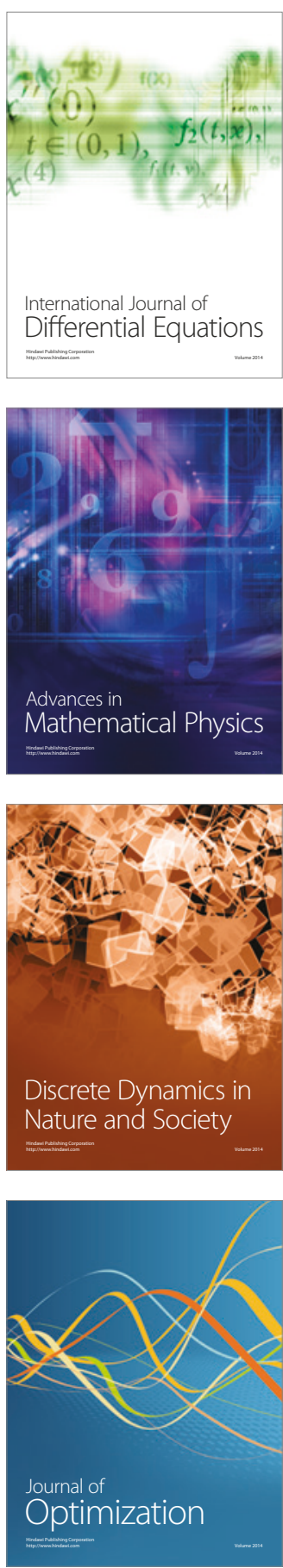